\title{
The Novel Role of LINC01235 in Metastasis of Gastric Cancer Cells by Inducing Epithelial- mesenchymal Transition
}

\section{Cheng Zhang}

Department of Gastroenterological Surgery, the Fourth Affiliated Hospital of China Medical University, Shenyang 110032, China https://orcid.org/0000-0001-5317-8775

\section{Yu Liang}

Department of Gastroenterological Surgery, the Fourth Affiliated Hospital of China Medical University, Shenyang 110032, China

\section{Chun-Dong Zhang}

Department of Gastroenterological Surgery, the Fourth Affiliated Hospital of China Medical University, Shenyang 110032, China

\section{Dong-Qiu Dai ( $\nabla$ daidq63@163.com )}

Department of Gastroenterological Surgery, the Fourth Affiliated Hospital of China Medical University, Shenyang 110032, China

\section{Research}

Keywords: gastric cancer, IncRNA, LINC01235, prognosis, epithelial-mesenchymal transition, metastasis

Posted Date: August 5th, 2020

DOl: https://doi.org/10.21203/rs.3.rs-50590/v1

License: (c) (1) This work is licensed under a Creative Commons Attribution 4.0 International License. Read Full License 


\section{Abstract}

\section{Background}

LncRNAs play a vital role in the tumorigenesis of gastric cancer (GC). The present study aims to explore the role of LINC01235 in clinical significance, prognostic prediction and GC metastasis.

\section{Methods}

We identified differentially expressed IncRNAs using stomach adenocarcinoma RNA-Seq data from The Cancer Genome Atlas (TCGA). The expression of LINC01235 in GC cell lines and tissues were confirmed by qRT-PCR assay. The overall survival analysis and univariate/ multivariate Cox regression analysis were performed to explore the prognostic value of LINC01235. In vitro assays were utilized to assess the effect of LINC01235 in cell migration and invasion. Western blotting measured the expression of EMT-induced proteins.

Results

This study determined that LINC01235 expression has a higher fold changes by analyzing TCGA RNASeq data. qRT-PCR assay confirmed that LINC01235 is significantly over-expressed in GC cells and tissues. Additionally, the overall survival analysis showed that patients with a higher LINC01235 expression had a poorer prognosis than those with a lower LINC01235 expression. Univariate Cox regression analysis indicated that high LINC01235 expression is positively correlated with poor prognosis. Moreover, LINC01235 was an independent poor prognostic marker for GC in multivariate Cox analysis. In vitro assays suggested that LINC01235 knockdown suppresses GC cell migration and invasion. GSEA revealed that high LINC01235 expression is strongly enriched in EMT pathway. Western blotting results revealed that LINC01235 silencing decreases the expression of EMT-induced proteins.

Conclusion

LINC01235 could promote GC cell metastasis via EMT and function as a prognostic biomarker.

\section{Background}

Cancer remains a major public health issue with an estimated 18.1 million cases and 9.6 million cancer deaths in 2018 worldwide ${ }^{1}$. Gastric cancer (GC) is a common malignant digestive tract tumor with a high incidence and mortality and a poor prognosis ${ }^{2,3}$. Even though GC incidence and mortality rates have been decreasing in the past few decades, there were still 1,033,701 new cases and 782,685 GC-related deaths around the world in $2018{ }^{4}$. More than $40 \%$ of GC patients are diagnosed with metastatic diseases and their response rate to chemotherapy and targeted therapy is unsatisfactory ${ }^{5,6}$. Therefore, GC is a common contributor to the worldwide cancer burden as measured by disability-adjusted life years lost ${ }^{7}$. 
The Human Genome Project found that protein-coding genes account for only $1 \%$ of the human genome, while the remaining genes have no protein-coding function ${ }^{8}$. The non-coding transcripts conclude housekeeping noncoding RNAs (for example: ribosomal, transfer, and small nuclear RNAs) and regulatory noncoding RNAs (for example: microRNAs and small interfering RNAs) ${ }^{9}$. Long non-coding RNAs (IncRNAs) with sequences $>200$ nucleotides in length have no protein-coding function and play vital biological roles in human cancers ${ }^{10}$. LncRNAs can be classified into five categories: sense, antisense, bidirectional, intronic, and intergenic ${ }^{9}$. The various molecular roles of IncRNAs vary for their different types.

Increasing evidence has indicated that IncRNAs are misregulated and participate in the development and progress of various cancers, including GC. For example, IncRNA HOTAIR promotes GC cell proliferation, migration, and invasion by sponging miR-331-3p and regulating HER2 expression ${ }^{11}$. Ectopic expression of HOTAIR also contributes to paclitaxel and doxorubicin resistance of GC cells by suppressing miR-217 expression ${ }^{12}$. Our previous study found that the DLX6-AS1/miR-204-5p/OCT1 positive feedback axis induces GC progression via EMT ${ }^{13}$. However, many vital biological roles of novel IncRNAs in GC need to be explored and verified.

The present study characterized stomach adenocarcinoma RNA-Seq data from The Cancer Genome Atlas (TCGA) and identified differentially expressed IncRNAs. LINC01235 expression was found to be of vital clinical significance. Further functional experiments confirmed that upregulated LINC01235 facilitates GC cell metastasis via EMT and may serve as a prognostic biomarker.

\section{Methods}

\section{Patients and samples}

A total of 36 paired GC and adjacent non-cancerous tissues ( $>5 \mathrm{~cm}$ from cancer tissue) used in the study were obtained from the Fourth Affiliated Hospital of China Medical University. All samples were confirmed by histopathological examination and were stored at $-80^{\circ} \mathrm{C}$ immediately after surgical resection. The study was authorized by the Research Ethics Committee of the Fourth Affiliated Hospital of China Medical University and informed consent was obtained from all patients.

\section{Cell Culture}

Human GC cell lines ((BGC-823, AGS, MGC-803, and SGC-7901) and normal gastric epithelial cell line (GES-1) were purchased from the Chinese Academy of Sciences (Shanghai, China). All cells were maintained in RPMI 1640 medium (HyClone, Logan, UT, USA) with $10 \%$ fetal bovine serum (Gibco, Grand Island, NY, USA), $100 \mathrm{U} / \mathrm{mL}$ penicillin, and $100 \mathrm{mg} / \mathrm{mL}$ streptomycin. The cells were incubated in humidified air at $37^{\circ} \mathrm{C}$ with $5 \% \mathrm{CO}_{2}$. 


\section{Rna Isolation And Quantitative Real-time Polymerase Chain Reaction (qrt-pcr)}

Total RNA samples were extracted from tissues and cell lines using RNAios Plus kits (TaKaRa Bio Technology, Dalian, China). RNA reverse transcription and quantitative PCR were performed with the ABI 7500 system (Applied Biosystems) using InRcute IncRNA First-Strand cDNA Synthesis kit and SYBR Green qPCR kit (TIANGEN, Beijing, China). The primers used for qRT-PCR were listed in Table 1. Relative gene expression was determined using the $2^{-\Delta \Delta C t}$ method.

\section{Cell Transfection}

Small interfering RNAs (siRNAs) designed and synthesized for decreasing gene expression by RiboBio were siRNA-LINC01235 \#1, \#2, \#3, and negative control siRNA-NC. The siRNA sequences are shown in Table 1. The siRNAs were transfected into GC cell lines (SGC-7901 and MGC-803) with riboFECT ${ }^{\mathrm{TM}} \mathrm{CP}$ transfection kit (RiboBio, Guangzhou, China) according to the manufacturer's protocol. Gene silencing effect was detected by qRT-PCR.

\section{Transwell Migration And Invasion Assay}

The numbers of SGC-7901 and MGC-803 cells were counted after transfection for $24 \mathrm{~h}$. Cell invasion and migration assays were carried out in 24-well plates with or without pre-coated Matrigel Basement Membrane Matrix (BD Biosciences). A total of $2.0 \times 10^{4}$ cells with $200 \mathrm{~mL}$ of FBS-free RPMI 1640 medium were plated into the upper chambers of the Transwell plates $(8.0-\mu \mathrm{m}$ pore size, Corning, New York, NY, USA). Lower chambers were filled with $500 \mathrm{~mL}$ of medium with $10 \% \mathrm{FBS}$. After a $24 \mathrm{~h}$ incubation, cells adhered to the lower surface of the upper chambers were fixed with $4 \%$ paraformaldehyde for $15 \mathrm{~min}$ and stained with $0.1 \%$ crystal violet (Solarbio) for $30 \mathrm{~min}$. Then, cell numbers per field of view were counted and photographed under a microscope.

\section{Western Blot Analysis}

The samples were lysed with RIPA buffer (Solarbio). Equivalent amounts of total protein were separated on $8 \%$ SDS-PAGE gels and transferred to PVDF membranes (Millipore, Billerica, MA, USA). The PVDF membranes were blocked with $5 \%$ skim milk for $2 \mathrm{~h}$ by gentle shaking. The primary antibodies were then incubated at $4{ }^{\circ} \mathrm{C}$ overnight according to the following dilution ratio: E-cadherin (1:400; Wanleibio), $\mathrm{N}$ cadherin (1:500), vimentin (1:500), Slug (1:1000), and $\beta$-actin (1:1000). The membranes were washed the following day and incubated with horseradish peroxidase-conjugated secondary antibody (goat antirabbit, 1:5000; Wanleibio). Finally, the signals were analyzed using enhanced chemiluminescence (ECL) substrate (Wanleibio) on a chemiluminescence detection system (Tanon, Shanghai, China). 


\section{Data Source And Bioinformatics Analysis}

The RNA-Seq data and clinical information for GC were obtained from the UCSC Xena platform ${ }^{14}$, which stores various datasets from TCGA. The IncRNA expression profiles were selected and used for screening differentially expressed IncRNAs with a limma package ${ }^{15}$ in R software. Relative LINC01235 expression values in GC and normal tissues were also collected from three microarray datasets (GSE65801, GSE51575, and GSE63089) and the GEPIA platform ${ }^{16}$. LINC01235 coding potential was calculated and predicted using CPC 2.0 project ${ }^{17}$. Gene set enrichment analysis (GSEA) was carried out to explore potential molecular functions that can be influenced by the LINC01235 expression level ${ }^{18}$. The hallmark gene sets and KEGG gene sets in the Molecular Signatures Database (MSigDB) were selected as the background during GSEA ${ }^{19}$.

\section{Statistical analysis}

The SPSS software V19.0 and GraphPad Prism 7.0 were used for statistical analysis. LINC01235 expression levels between groups were analyzed by paired or unpaired test. Overall survival analysis was performed using the Kaplan-Meier method. The univariate and multivariate Cox regression analyses were used to test the effects of variables on survival. Pearson correlation analysis was utilized for calculating the expression correlation between genes. $P$-values $<0.05$ were considered statistically significant.

\section{Results}

\section{LINC01235 is up-regulated in GC}

The IncRNA expression profiles, including $375 \mathrm{GC}$ tissues and 32 adjacent normal tissues, were utilized for identifying differentially expressed IncRNAs. According to the cut-off criterion of $|F C|>2.0$ (fold change) and adjusted $P$-value $<0.05$, there are 1537 up-regulated and 329 down-regulated IncRNAs in GC compared to adjacent normal tissues (Fig. 1A). LINC01235 had a log2FC value of 3.1 and was significantly up-regulated in GC tissues from the TCGA data (Fig. 1B). The retrieved microarray datasets from the Gene Expression Omnibus (GEO) repository showed that LINC01235 is significantly up-regulated in GC tissues compared to corresponding normal tissues (Fig. 1C). In addition, qRT-PCR was used to confirm the aberrant expression of LINC01235. In line with the above results, LINC01235 was frequently overexpressed in GC tissues and cell lines (Fig. 1D, E). LINC01235 was found to have a biased expression in multiple human organs, including brain, thyroid, esophagus, breast, stomach, and colon (Fig. 2A). LINC01235 coding capacity was also investigated. The prediction indicated that LINC01235 has a coding probability of 0.058001 and belongs to noncoding genes (Fig. 2B).

\section{Clinical Significance Of Linc01235 In Gc}


The aberrant expression of LINC01235 in patients with or without metastasis was investigated to explore its significance in metastasis. The results suggested that patients with metastasis have a higher LINC01235 expression level than those without metastasis (Fig. 3A). The GC patients with detailed survival time and status data were included in the Kaplan-Meier survival analysis. A total of 352 patients were sorted into high or low expression groups according to their LINC01235 median expression value. The results showed that patients with a high LINC01235 expression have a poorer survival probability than those with a low LINC01235 expression (Fig. 3B). Univariate Cox regression analysis demonstrated that pathologic $\mathrm{T}, \mathrm{N}$, and M stages, tumor stage, and LINC01235 expression are significantly associated with patients' overall survival. Further multivariate Cox regression analysis indicated that pathologic $\mathrm{M}$ stage and LINC01235 expression can be independent prognostic factors in GC patients (Fig. 3C).

\section{Linc01235 Knockdown Attenuates Gc Cell Migration And Invasion}

SGC-7901 and MGC-803 cells were transfected with siRNA to silence LINC01235 expression and investigate the biological role of LINC01235. After a 24-h transfection, qRT-PCR confirmed the validity of the knockdown effect of the three siRNAs (Fig. 4A). Transwell experiments demonstrated that LINC01235 silencing significantly decreased the migration and invasion ability of SGC-7901 and MGC-803 cells (Fig. 4B).

\section{Linc01235 Participates In Gc Cell Emt}

GSEA was performed in order to explore potential biological processes involving LINC01235. According to the median value of LINC01235 expression, a total of $375 \mathrm{GC}$ patients were classified into two categories (high vs. low). The findings showed that high LINC01235 expression is significantly enriched in EMT, angiogenesis, and focal adhesions (Fig. 5A). Expression correlation between LINC01235 and EMTassociated genes was also analyzed. Pearson correlation analysis indicated that LINC01235 expression is negatively correlated with $\mathrm{CDH} 1(r=-0.27, P<0.01)$ and positively correlated with VIM $(r=0.53, P<$ $0.01)$, SNAI2 $(r=0.49, P<0.01)$, and CDH2 $(r=0.27, P<0.01$, Fig. 5B). Pearson correlation results for additional EMT-associated genes are shown in Supplementary Fig. 1. Furthermore, western blotting analysis demonstrated that silencing LINC01235 can increase E-cadherin (epithelial marker) expression and decrease N-cadherin, vimentin, and Slug (mesenchymal markers) expression (Fig. 5C). Thus, LIN01235 may promote GC cell migration and invasion via EMT.

\section{Discussion}

The higher incidence and mortality of GC are attributed to the fact that most patients are diagnosed at an advanced stage, thereby losing their opportunity for radical surgery. Research has shown that the fiveyear survival rate of patients with advanced GC is $<20 \% 20,21$. Increasing numbers of studies have found 
that IncRNAs can act as critical regulators in GC metastasis and tumor growth, potentially becoming promising targets for GC treatment ${ }^{22-24}$. LINC01235 is a large intergenic noncoding RNA that is located on human chromosome 9p23 and has three exons. RNA-Seq data from the Human Protein Atlas indicated that LINC01235 has a biased expression level in human tissues ${ }^{25}$. However, the role and function of LINC01235 in GC remain unclear.

The present study identified differentially expressed IncRNAs in GC and normal tissues by analyzing RNASeq data from the TCGA database. LINC01235 was found to have a log2FC value of 3.1 in cancer tissues compared to normal tissues. The GEO datasets were also explored. The results of three GC microarray datasets revealed that LINC01235 is significantly up-regulated in cancer tissues compared to paired normal tissues. Then, qRT-PCR results confirmed that LINC01235 is over-expressed in GC cell lines and tissues. All of these findings support the aberrant expression of LINC01235 in GC. Therefore, LINC01235 may act as a novel proto-oncogene in the occurrence and progression of GC.

Furthermore, LINC01235 has a higher level in GC patients with metastasis than in those without. KaplanMeier survival analysis illustrated that patients with a high LINC01235 expression have a poorer survival probability than those with a low LINC01235 expression $(P=0.0017)$. Univariate Cox regression analysis indicated that high LINC01235 expression is positively correlated with poor prognosis $(\mathrm{HR}=1.49,95 \% \mathrm{Cl}$ [1.06-2.10], $P=0.021$ ). Furthermore, LINC01235 is an independent poor prognostic marker for GC in multivariate Cox analysis ( $\mathrm{HR}=1.48,95 \% \mathrm{Cl}[1.04-2.09], P=0.029)$. To the best of our knowledge, no previous studies have investigated LINC01235 expression and biological role in GC. The function of LINC01235 in GC cells was explored using loss-of-function assay. The results revealed that LINC01235 silencing significantly decreases the migration and invasion capacity of GC cells. The above results indicated that LINC01235 is of vital clinical significance and may be a potential therapeutic target for metastatic GC.

Tumor metastasis is a multi-step process that includes migration and invasion of epithelial-derived tumor cells, blood circulation, evasion from blood vessels, and colonization of new microenvironments ${ }^{26,27}$. GSEA was performed to explore potential biological processes involving LINC01235. LINC01235 expression was significantly enriched in EMT, angiogenesis, and focal adhesions. EMT, a biological process in which tumor cells lose their epithelial polarity and obtain mesenchymal characteristics, has been found to play a vital role in the initiation of metastasis ${ }^{28,29}$. Furthermore, expression correlation between LINC01235 and EMT-associated genes was analyzed. Pearson correlation analysis indicated that LINC01235 expression is negatively correlated with $\mathrm{CDH} 1(r=-0.27, P<0.01)$ and positively correlated with VIM $(r=0.53, P<0.01)$, SNAI2 $(r=0.49, P<0.01)$, and CDH2 $(r=0.27, P<0.01)$. Western blotting assays confirmed that LINC01235 silencing can increase E-cadherin expression and decrease Ncadherin, vimentin, and Slug expression. These findings showed that LINC01235 may promote GC cell migration and invasion via EMT.

\section{Conclusions}


The present study found that IncRNA LINC01235 is up-regulated in GC cells and tissues, underscoring its vital clinical significance. Loss-of-function experiments demonstrated that upregulated LINC01235 promotes GC cell metastasis via EMT and may be a valuable prognostic biomarker and treatment target for metastatic GC. However, the in-depth mechanism by which LINC01235 facilitates GC cell migration and invasion requires more investigation in future studies.

\section{Declarations}

\section{Ethics approval and consent to participate}

The study was authorized by the Research Ethics Committee of the Fourth Affiliated Hospital of China Medical University and informed consent was obtained from all patients.

\section{Consent for publication}

Not applicable.

\section{Availability of data and materials}

The datasets used and/or analysed during the current study are available from the corresponding author on reasonable request.

\section{Competing interests}

The authors declare that they have no competing interests.

\section{Funding}

This work was supported by the National Natural Science Foundation of China (No. 81972322), and the China Scholarship Council (No. 201908050148).

\section{Authors' contributions}

Dai DQ and Zhang C designed the study; Zhang C, Zhang CD and Liang Y performed data processing and analysis; Zhang $\mathrm{C}$ wrote the paper. All authors participated in revising the final manuscript and approved it for publication.

\section{Acknowledgements}

Not applicable. 


\section{References}

1. Han RL, Wang FP, Zhang PA, Zhou XY, Li Y. miR-383 inhibits ovarian cancer cell proliferation, invasion and aerobic glycolysis by targeting LDHA. Neoplasma. 2017;64:244-52.

2. Bray F, Ferlay J, Soerjomataram I, Siegel RL, Torre LA, Jemal A. Global cancer statistics 2018 : GLOBOCAN estimates of incidence and mortality worldwide for 36 cancers in 185 countries. CA Cancer J Clin. 2018;68:394-424.

3. Chen W, Sun K, Zheng R, et al. Cancer incidence and mortality in China, 2014. Chin J Cancer Res. 2018;30:1-12.

4. Thrift AP, El-Serag HB. Burden of Gastric Cancer. Clin Gastroenterol Hepatol. 2019.

5. Koizumi W, Kim YH, Fujii M, et al. Addition of docetaxel to S-1 without platinum prolongs survival of patients with advanced gastric cancer: a randomized study (START). J Cancer Res Clin Oncol. 2014;140:319-28.

6. Bernards N, Creemers GJ, Nieuwenhuijzen GA, Bosscha K, Pruijt JF, Lemmens VE. No improvement in median survival for patients with metastatic gastric cancer despite increased use of chemotherapy. Ann Oncol. 2013;24:3056-60.

7. Global Burden of Disease. Cancer C, Fitzmaurice C, Abate D, et al. Global, Regional, and National Cancer Incidence, Mortality, Years of Life Lost, Years Lived With Disability, and Disability-Adjusted Life-Years for 29 Cancer Groups, 1990 to 2017: A Systematic Analysis for the Global Burden of Disease Study. JAMA Oncol. 2019.

8. Schuler GD, Boguski MS, Stewart EA, et al. A gene map of the human genome. Science. 1996;274:540-6.

9. Ponting CP, Oliver PL, Reik W. Evolution and functions of long noncoding RNAs. Cell. 2009;136:62941.

10. Rinn JL, Chang HY. Genome regulation by long noncoding RNAs. Annu Rev Biochem. 2012;81:14566.

11. Liu XH, Sun M, Nie FQ, et al. Lnc RNA HOTAIR functions as a competing endogenous RNA to regulate HER2 expression by sponging miR-331-3p in gastric cancer. Mol Cancer. 2014;13:92.

12. Wang H, Qin R, Guan A, et al. HOTAIR enhanced paclitaxel and doxorubicin resistance in gastric cancer cells partly through inhibiting miR-217 expression. J Cell Biochem. 2018;119:7226-34.

13. Liang Y, Zhang CD, Zhang C, Dai DQ. DLX6-AS1/miR-204-5p/OCT1 positive feedback loop promotes tumor progression and epithelial-mesenchymal transition in gastric cancer. Gastric Cancer. 2019.

14. Goldman M, Craft B, Hastie M, et al. The UCSC Xena platform for public and private cancer genomics data visualization and interpretation. 2019: 326470.

15. Ritchie ME, Phipson B, Wu D, et al. limma powers differential expression analyses for RNAsequencing and microarray studies. Nucleic Acids Res. 2015;43:e47.

16. Tang Z, Kang B, Li C, Chen T, Zhang Z. GEPIA2: an enhanced web server for large-scale expression profiling and interactive analysis. Nucleic Acids Res. 2019;47:W556-60. 
17. Kang YJ, Yang DC, Kong L, et al. CPC2: a fast and accurate coding potential calculator based on sequence intrinsic features. Nucleic Acids Res. 2017;45:W12-6.

18. Subramanian A, Tamayo P, Mootha VK, et al. Gene set enrichment analysis: a knowledge-based approach for interpreting genome-wide expression profiles. Proc Natl Acad Sci U S A. 2005;102:15545-50.

19. Liberzon A, Birger C, Thorvaldsdottir H, Ghandi M, Mesirov JP, Tamayo P. The Molecular Signatures Database (MSigDB) hallmark gene set collection. Cell Syst. 2015;1:417-25.

20. Suzuki H, Oda I, Abe S, et al. High rate of 5-year survival among patients with early gastric cancer undergoing curative endoscopic submucosal dissection. Gastric Cancer. 2016;19:198-205.

21. Kim YI, Kim YW, Choi IJ, et al. Long-term survival after endoscopic resection versus surgery in early gastric cancers. Endoscopy. 2015;47:293-301.

22. Wang X, Liang Q, Zhang L, et al. C8orf76 Promotes Gastric Tumorigenicity and Metastasis by Directly Inducing IncRNA DUSP5P1 and Associates with Patient Outcomes. Clin Cancer Res. 2019;25:3128-40.

23. Zheng J, Zhang H, Ma R, Liu H, Gao P. Long non-coding RNA KRT19P3 suppresses proliferation and metastasis through COPS7A-mediated NF-kappaB pathway in gastric cancer. Oncogene. 2019;38:7073-88.

24. Hauptman N, Glavac D. MicroRNAs and long non-coding RNAs: prospects in diagnostics and therapy of cancer. Radiol Oncol. 2013;47:311-8.

25. Fagerberg L, Hallstrom BM, Oksvold P, et al. Analysis of the human tissue-specific expression by genome-wide integration of transcriptomics and antibody-based proteomics. Mol Cell Proteomics. 2014;13:397-406.

26. Wen J, Min X, Shen M, et al. ACLY facilitates colon cancer cell metastasis by CTNNB1. J Exp Clin Cancer Res. 2019;38:401.

27. Liu Y, Cao X. Characteristics and Significance of the Pre-metastatic Niche. Cancer Cell. 2016;30:66881.

28. Mani SA, Guo W, Liao MJ, et al. The epithelial-mesenchymal transition generates cells with properties of stem cells. Cell. 2008;133:704-15.

29. Peng Z, Wang CX, Fang EH, Wang GB, Tong Q. Role of epithelial-mesenchymal transition in gastric cancer initiation and progression. World J Gastroenterol. 2014;20:5403-10.

\section{Figures}


A

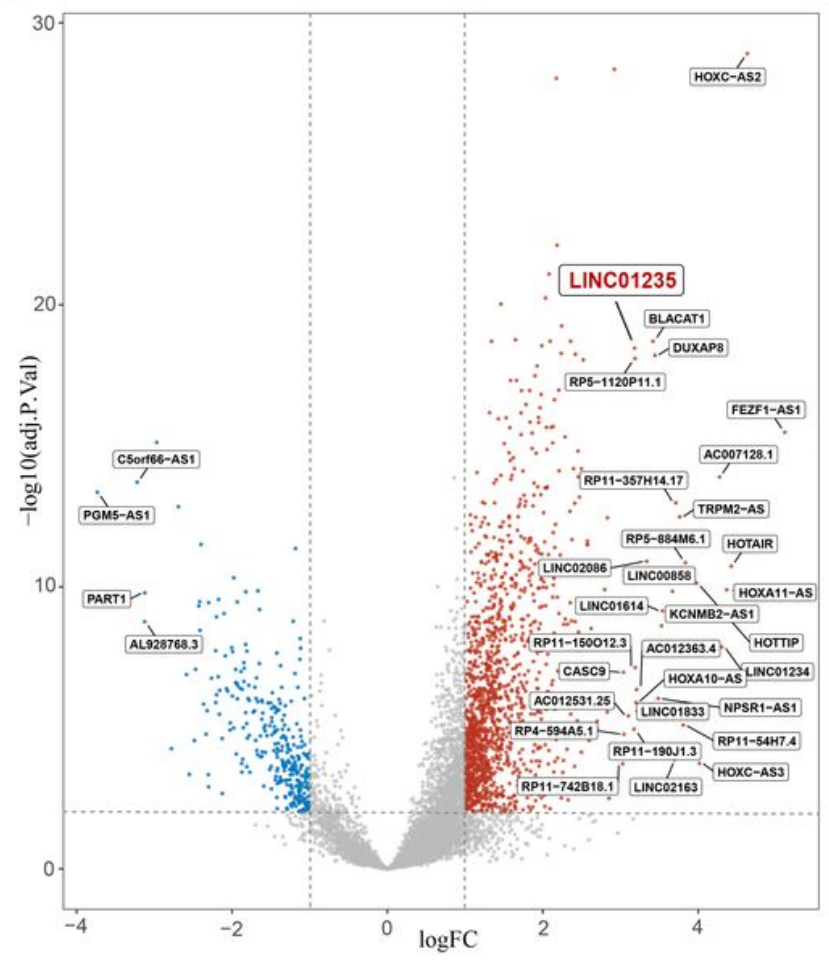

$\mathrm{B}$

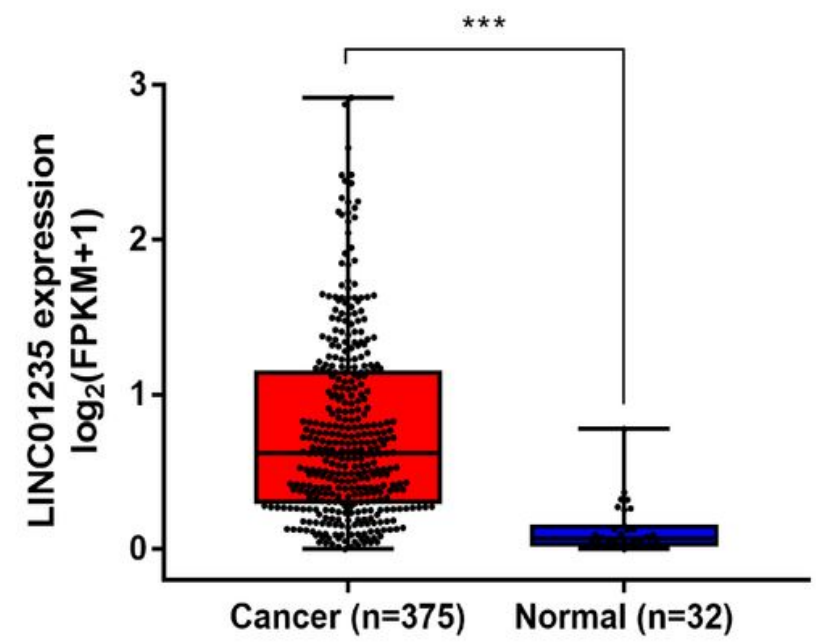

$\mathrm{C}$

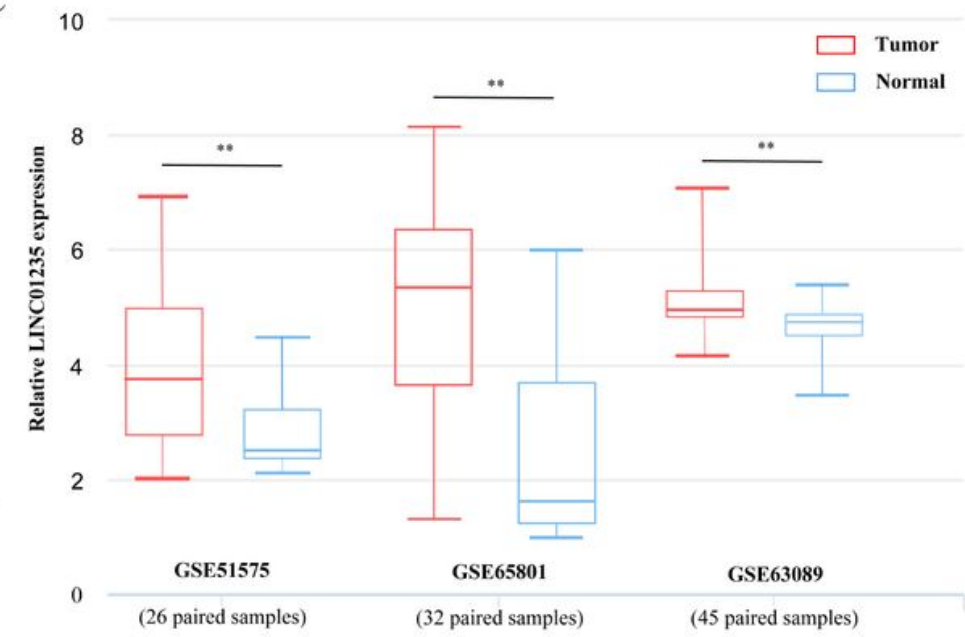

D

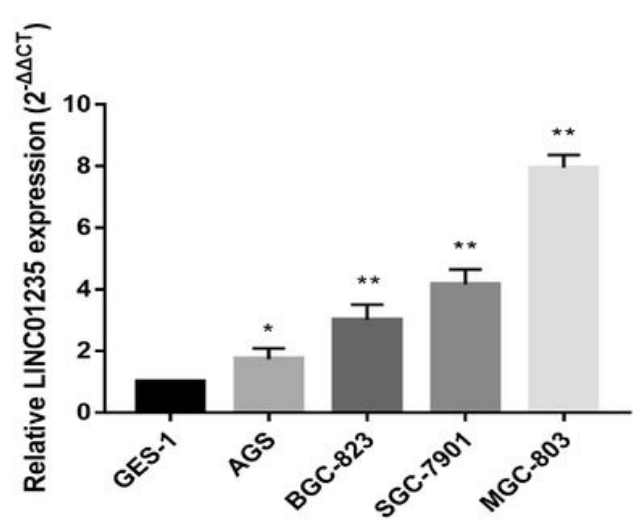

E

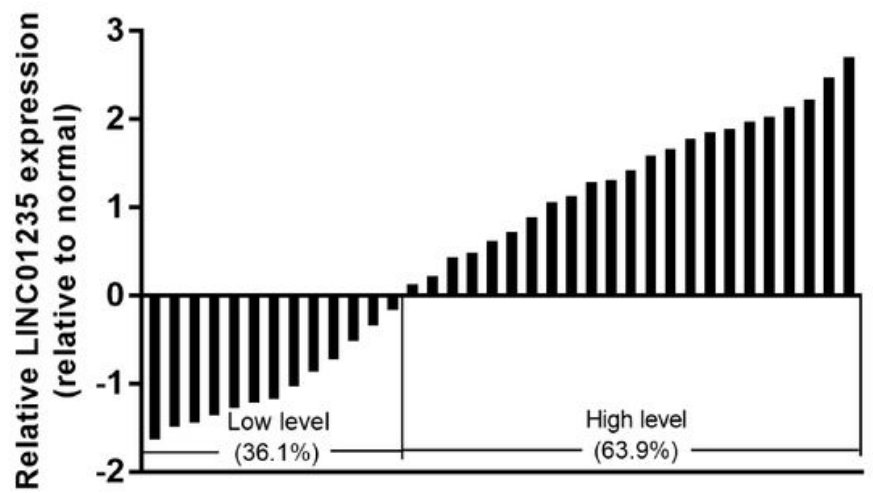

Figure 1

LINC01235 expression in GC cells and tissues. (A) Volcano plot represents differentially expressed IncRNAs that were identified by RNA-Seq data. Blue, grey, and red points represent down-regulated, not differentially expressed, and up-regulated IncRNAs, respectively. (B) Relative LINC01235 expression scatter plots in $372 \mathrm{GC}$ and 32 adjacent normal tissues. (C) Relative LINC01235 expression in paired GC tissues from three GEO datasets. (D) qRT-PCR results revealed that LINC01235 is up-regulated in GC cells and tissues. ${ }^{\star} \mathrm{P}<0.05,{ }^{\star *} \mathrm{P}<0.01$, and ${ }^{\star \star *} \mathrm{P}<0.001$. 
The median expression of tumor and normal samples in bodymap
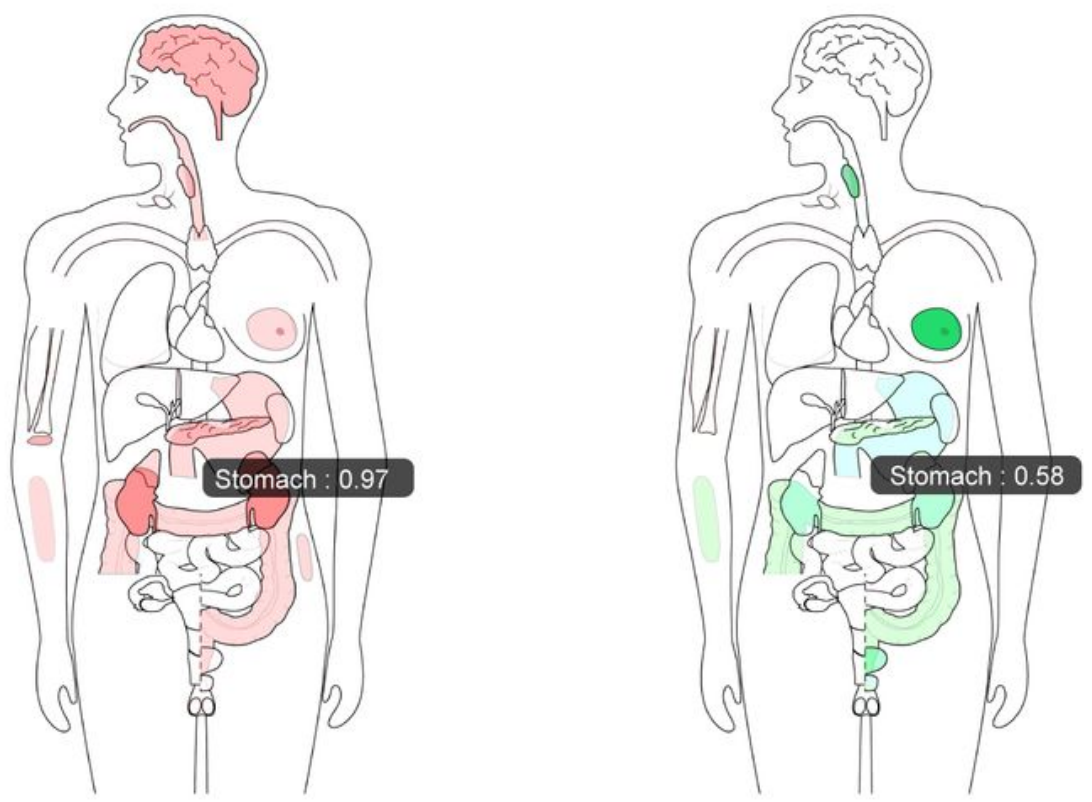

$\log _{2}(T P M+1)$ Scale

B PUTATIVE PEPTIDE LENGTH (LOG10)

0.25
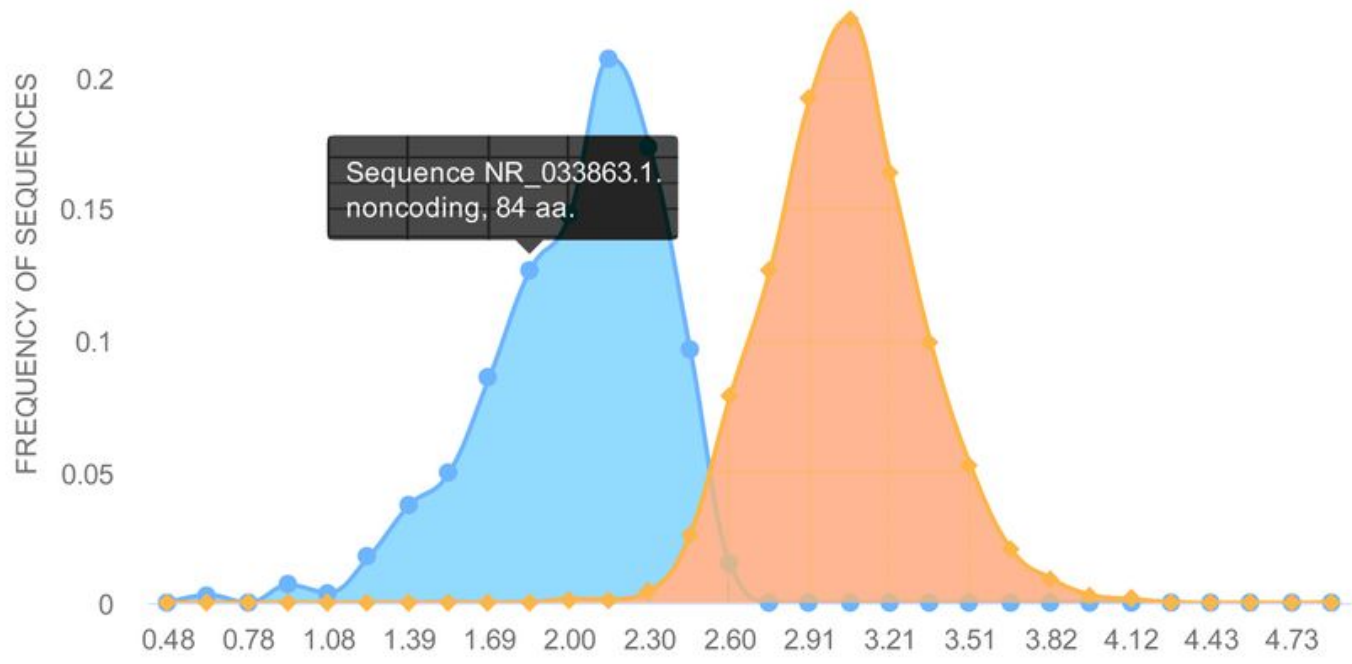

$\begin{array}{lllllllllllllll}0.48 & 0.78 & 1.08 & 1.39 & 1.69 & 2.00 & 2.30 & 2.60 & 2.91 & 3.21 & 3.51 & 3.82 & 4.12 & 4.43 & 4.73\end{array}$

Noncoding Coding

\begin{tabular}{|l|l|l|l|l|l|l|l|}
\hline ID & Label & Coding probability & Peptide length(as) & Fickett score & Isoelectric point & ORF integrity & Detalls \\
\hline LINC01235 & noncoding & 0.058001 & 84 & 0.2895 & 10.006652832 & complete & Vew
\end{tabular}

\section{Figure 2}

LINC01235 expression distribution in human body and its coding probability prediction. (A) LINC01235 is differentially expressed in multiple human organs, including brain, thyroid, esophagus, breast, stomach, and colon. (B) LINC01235 is predicted to have no protein coding capacity. 

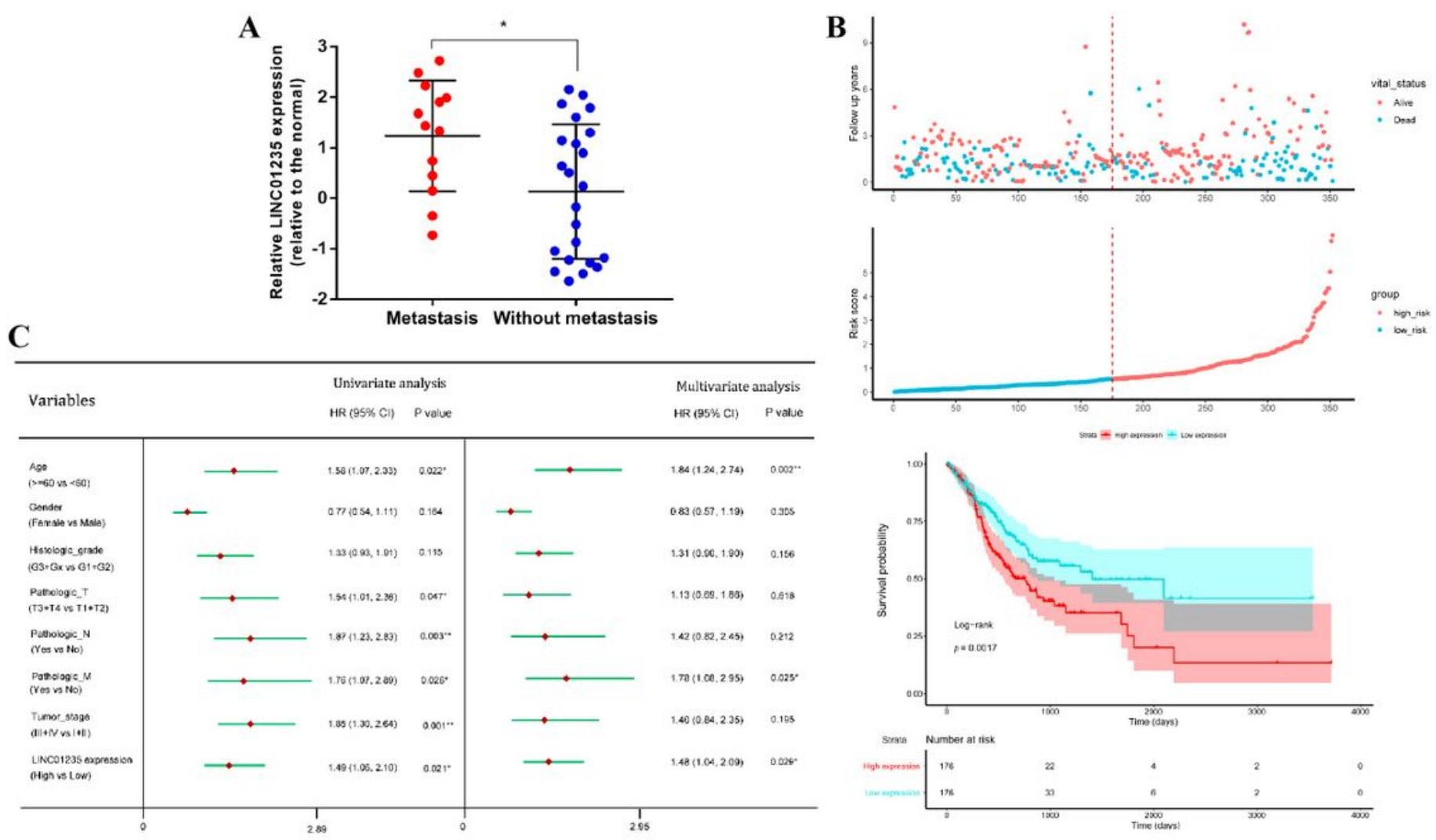

\section{Figure 3}

Clinical significances of LINC01235 in GC. (A) Patients with metastasis have higher LINC01235 expression level than those without metastasis. (B) Overall survival analysis showed that patients with higher LINC01235 expression have poorer prognosis than those with lower LINC01235 expression ( $P=$ 0.0017). (C) Univariate and multivariate Cox analysis indicated that LINC01235 is an independent poor prognostic marker for GC. ${ }^{*} \mathrm{P}<0.05$, ${ }^{\star *} \mathrm{P}<0.01$, and ${ }^{\star \star \star} \mathrm{P}<0.001$. 
A

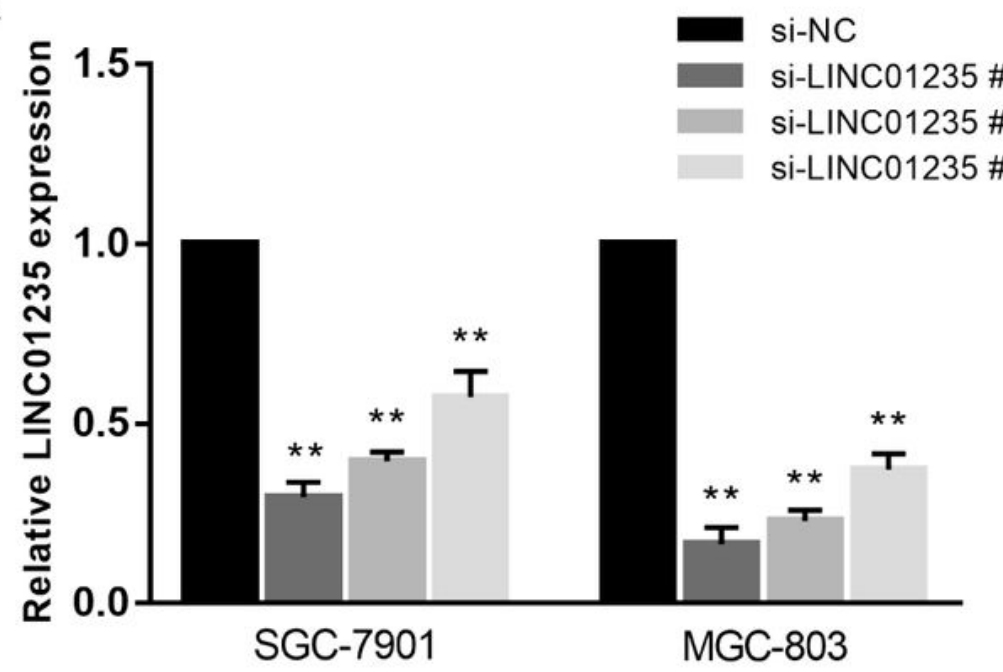

B
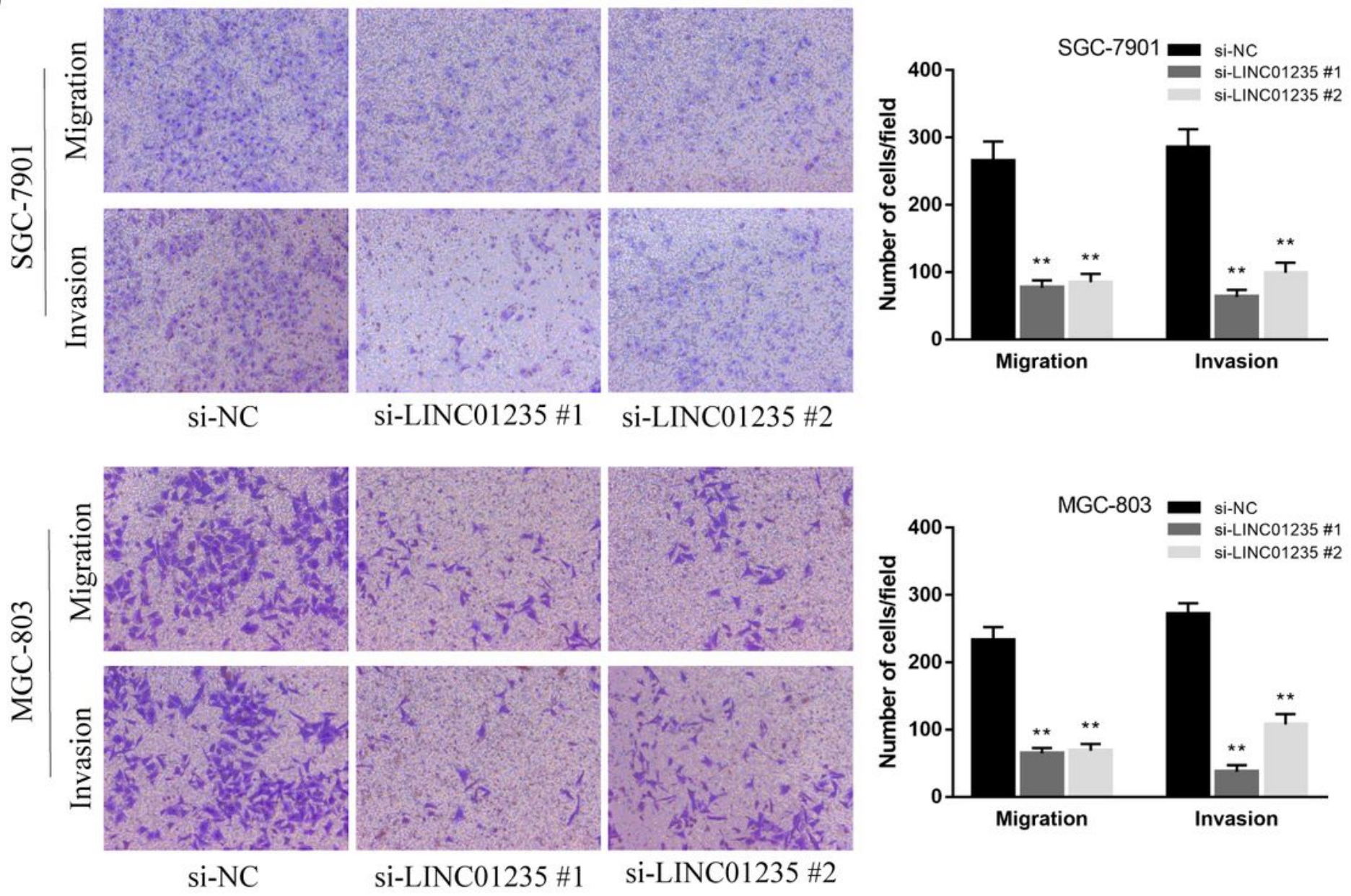

Figure 4

LINC01235 migration and invasion ability in GC. (A) LINC01235 knockdown effect was detected by qRTPCR. (B) LINC01235 silencing can significantly weaken GC cell migration and invasion capacity. ${ }^{*} \mathrm{r}$ $0.05, * \star P<0.01$, and $* \star * P<0.001$. 
A
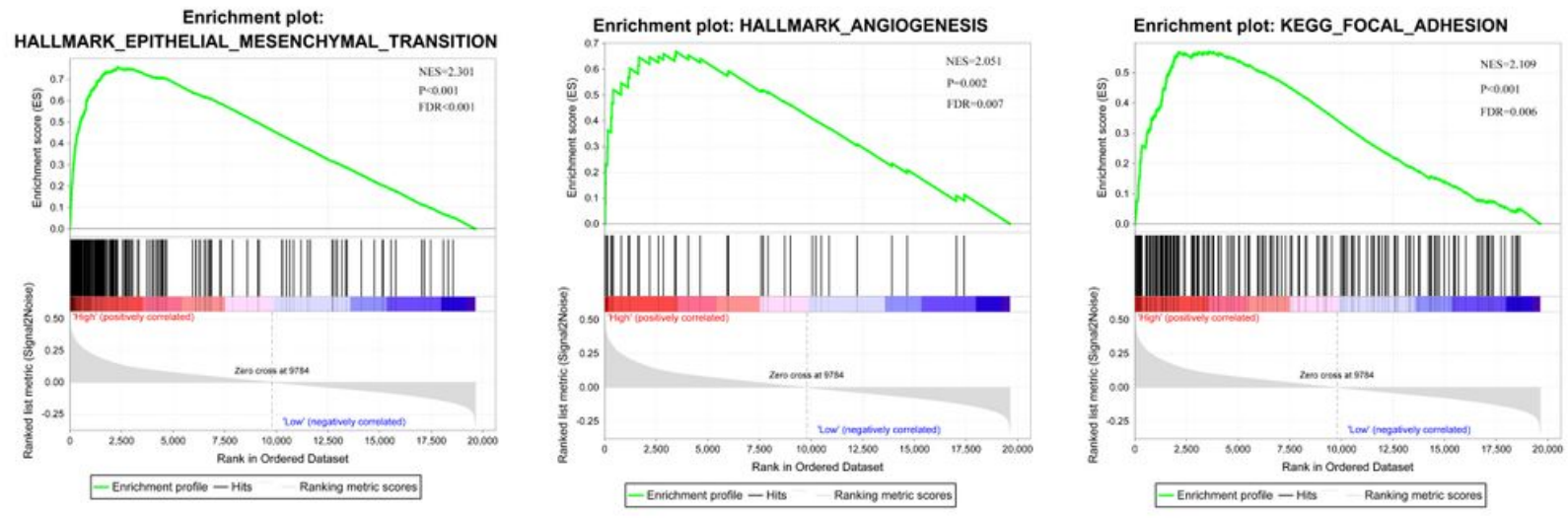

B

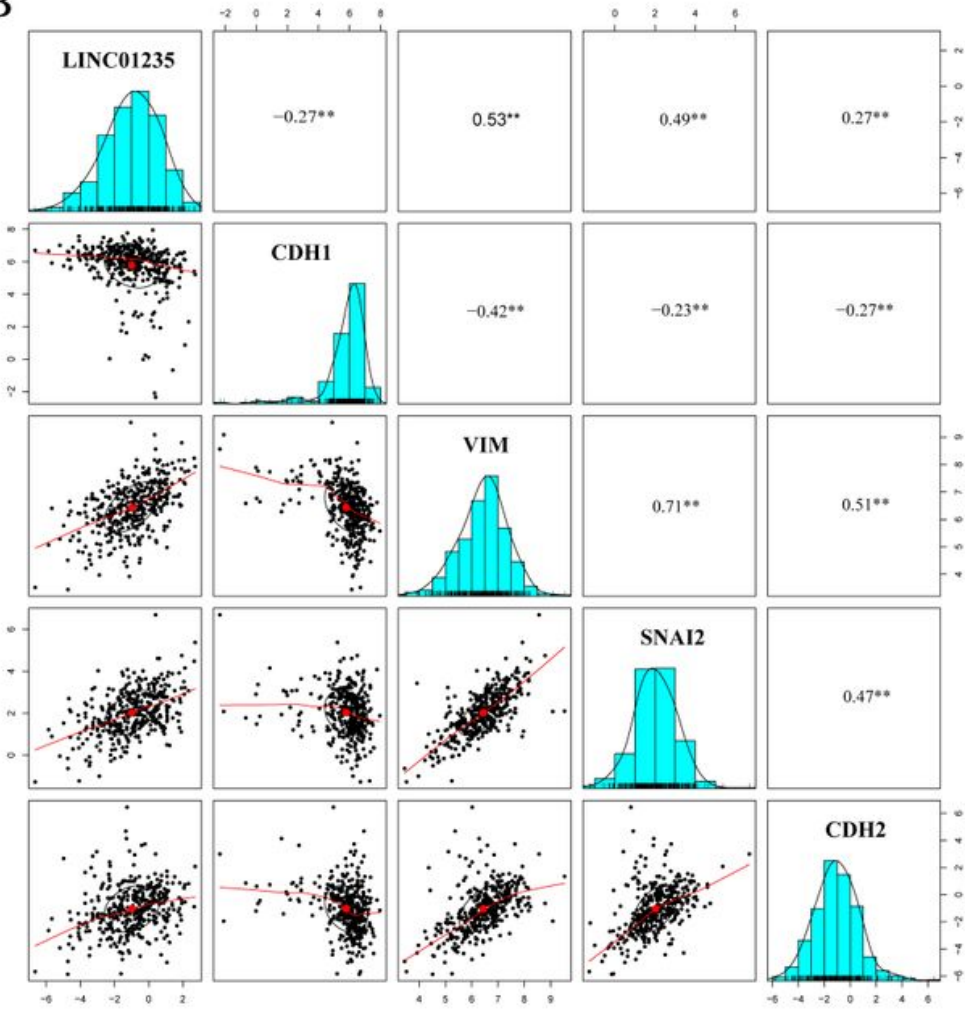

$\mathrm{C}$

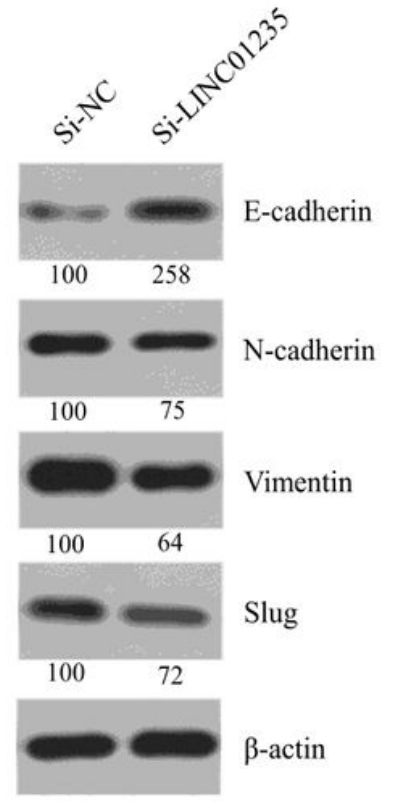

SGC-7901

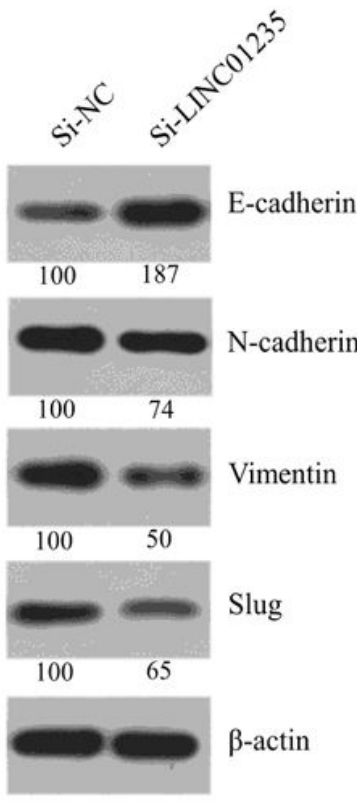

MGC-803

\section{Figure 5}

Oncogene role of LINC01235 in EMT regulation. (A) GSEA revealed that LINC01235 participates in EMT, angiogenesis, and focal adhesions. (B) Pearson correlation analysis indicated that LINC01235 expression is negatively correlated with $\mathrm{CDH} 1(\mathrm{r}=-0.27, \mathrm{P}<0.01)$ and positively correlated with $\mathrm{VIM}(r=0.53, \mathrm{P}<$ $0.01)$, SNAI2 ( $r=0.49, \mathrm{P}<0.01)$, and $\mathrm{CDH} 2(r=0.27, \mathrm{P}<0.01)$. (C) Western blotting assay demonstrated that LINC01235 silencing can increase E-cadherin expression and decrease $\mathrm{N}$-cadherin, vimentin, and Slug expression. ${ }^{*} \mathrm{P}<0.05,{ }^{*} \mathrm{P}<0.01$, and ${ }^{\star * *} \mathrm{P}<0.001$.

\section{Supplementary Files}

This is a list of supplementary files associated with this preprint. Click to download. 
- SupplementaryFigure1.tif

- SupplementaryFigure1.tif

- SupplementaryFigure1.tif 